\title{
Tokamak Magnetohydrodynamic Equilibrium States with Axisymmetric Boundary and a 3D Helical Core
}

\author{
W. A. Cooper,* J. P. Graves, A. Pochelon, O. Sauter, and L. Villard \\ Ecole Polytechnique Fédérale de Lausanne (EPFL), Centre de Recherches en Physique des Plasmas, \\ Association Euratom-Confédération Suisse, CH-1015 Lausanne, Switzerland
}

(Received 21 April 2010; published 16 July 2010)

\begin{abstract}
Magnetohydrodynamic (MHD) equilibrium states with imposed axisymmetric boundary are computed in which a spontaneous bifurcation develops to produce an internal three-dimensional (3D) configuration with a helical structure in addition to the standard axisymmetric system. Equilibrium states with similar MHD energy levels are shown to develop very different geometric structures. The helical equilibrium states resemble saturated internal kink mode structures.
\end{abstract}

DOI: 10.1103/PhysRevLett.105.035003

The essential confinement of particles and energy in magnetically enclosed plasmas is described by magnetohydrodynamics (MHD). The tokamak is the leading fusion energy research concept in which the plasma is expected to be contained in an essentially axisymmetric configuration with relatively small ripple effects from the finite toroidal coils. Symmetry of the equilibrium state along the toroidal coordinate grants the tokamak many appealing properties such as toroidal mass flow, which is known to reduce MHD instability and reduce non-MHD transport of particles and energy, as well as important conservation properties of single particles, thus enhancing confinement on various scales.

It is shown in this Letter that despite imposing axisymmetry (toroidal symmetry) at the edge of the plasma, enforced by the geometry of the coil system, the preferred lowest energy state of MHD equilibrium can be nonaxisymmetric in the plasma center. The computation of threedimensional (3D) helical cores constitutes a paradigm shift for the description of tokamak equilibria that opens the way for the application of theoretical and simulation tools developed for stellarator analysis of MHD stability, guiding center particle orbits, kinetic stability, wave propagation or heating, neoclassical transport, gyrokinetics, etc., to determine the impact of these novel 3D states on a large range of magnetic confinement physics phenomena.

The ignorable toroidal angle coordinate in axisymmetric magnetic confinement systems allows a simplified description of the MHD equilibrium state through the GradShafranov equation [1,2]. A more sophisticated approach invokes the minimization of the MHD energy to achieve an equilibrium state which can be naturally extended to model 3D systems with the imposition of nested magnetic flux surfaces and a single magnetic axis [3-9]. Tokamak devices, though nominally axisymmetric, display internal plasma reorganization phenomena that can break the symmetry of the system. In the tokamak à configuration variable (TCV) [10], a transition is observed where core sawteeth relaxations are replaced by global oscillations with low poloidal and toroidal mode numbers $[11,12]$. In
PACS numbers: 52.55.Fa, 52.35.Py, 52.55.Hc, 52.55.Tn

these discharges, the inverse rotational transform $q$ profiles are nearly flat or slightly reversed. One possible explanation for this transition is that $q_{\min }$, the minimum value of safety factor $q$ within the plasma, becomes greater than unity. Equilibria with $q_{\min }$ near unity are of interest in the present contribution. Similarly saturated ideal modes in the MAST device have also been recently reported [13]. The "snake" structures in JET constitute another symmetrybreaking internal structure triggered by pellet injection. A theoretical interpretation proposed dealt with pellet deposition in a magnetic island localized at the $q=1$ surface [14]. The reversed field experiment-mod (RFX-mod) device has detected helical internal structures when the current is sufficiently large that quasi-single-helicity (QSH) structures with a $m=1, n=7$ periodicity form that correspond to the helicity of the most internally resonant mode of an axisymmetric configuration [15]. These were successfully modeled with helical MHD equilibrium states computed with the VMEC code [8]. Bifurcated equilibria in a straight Spheromak have also been calculated [16].

Theoretical applications have concentrated on nonlinear stability evolution and saturation mechanisms to describe tokamak plasmas. Nonlinear quasi-interchange [17] and internal kinkmodes, that are evolving slowly $[18,19]$, have been calculated with hollow and flat $q$ profiles with $q \geq 1$ everywhere. Nonlinearly stable states have also been determined with the BETAS code [20]. An axisymmetric equilibrium is computed followed by a second minimization for stability with a linear constraint imposed. A nonlinearly stable solution is finally obtained which differs from the original (axisymmetric in the case of tokamak studies) equilibrium [21,22]. Solutions were found for tokamaks and stellarators that correspond to low order ballooning-type modes that do not alter the original magnetic axis position.

Energy minimization schemes to determine the equilibrium state are based on the first variation of

$$
\mu_{0} W=\iiint d^{3} x\left(\frac{B^{2}}{2}+\frac{\mu_{0} p_{\|}}{\Gamma-1}\right)
$$



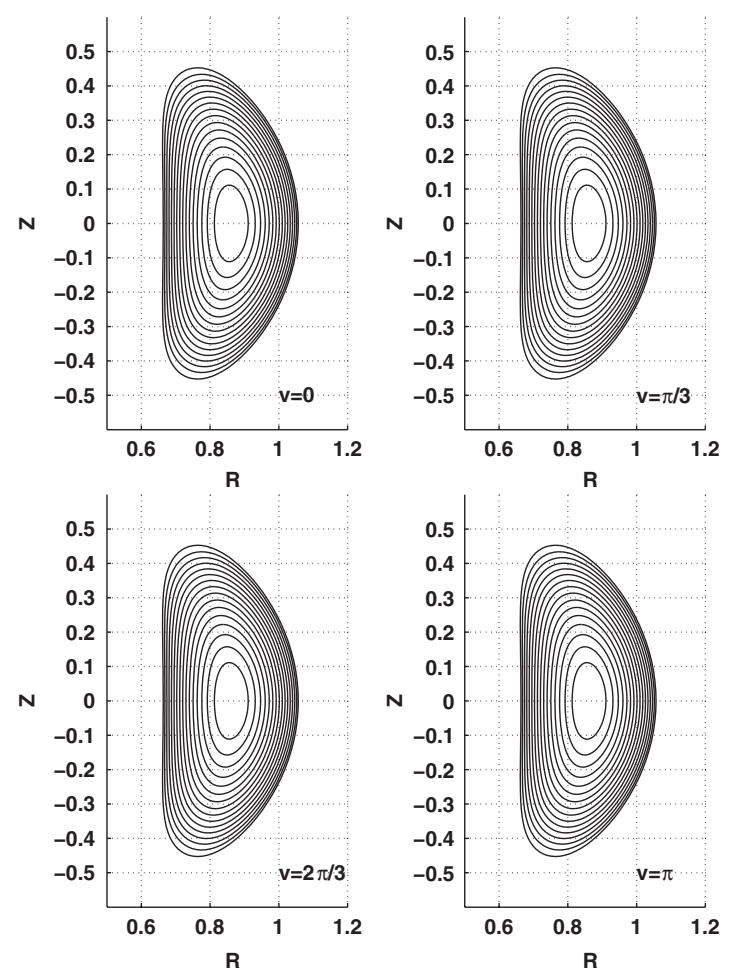

FIG. 1. Toroidal magnetic flux surface contours for the axisymmetric equilibrium state branch at four different toroidal cross sections with angles $v=0, \pi / 3,2 \pi / 3$, and $\pi$ in TCVlike axisymmetric boundary geometry with elongation 2.4 , triangularity 0.3 , and rotational transform profile $\iota(s)=$ $0.9+0.2 s-0.8 s^{6}$. The equilibrium energy is $\mu_{0} W_{\text {axi }}=$ 1.454968 .

where $W$ is the MHD energy, $B$ is the magnetic field strength, $p_{\|}$is the plasma pressure parallel to the magnetic field lines (for isotropic pressure conditions $p_{\|} \rightarrow p$ ), $\mu_{0}$ is the permeability of free space and $\Gamma$ is the ratio of specific heats. In this study, we have used $\Gamma=0$ and limited the calculations to fixed boundary conditions. Specifically, an axisymmetric elongated $\mathrm{D}$-shaped boundary was chosen that approximates a configuration readily achievable in the TCV tokamak [10]. The plasma-vacuum interface is described by the Fourier expansion

$$
R_{b}=R_{0}+0.2 \cos u+0.06 \cos 2 u, \quad Z_{b}=0.48 \sin u,
$$

where $u$ is the poloidal angle, $R_{b}$ is the distance of a plasma boundary point from the major axis and $Z_{b}$ is the distance from the midplane. We employ MKS SI units in this work. For TCV, we take $R_{0}=0.8 \mathrm{~m}$. Two profiles must be specified to obtain a MHD equilibrium with scalar plasma pressure. For simplicity, we prescribe initially the pressure as $p(s)=p_{0}(1-s)$, where $0 \leq s \leq 1$ is the radial variable proportional to the normalized toroidal magnetic flux (the flux divided by its value at the plasma boundary). We choose $p_{0}$ such that the volume-averaged plasma $\langle\beta\rangle \simeq$ $0.5 \%$. The second profile we designate is that corresponding to the rotational transform $\iota(s)=1 / q(s)$.
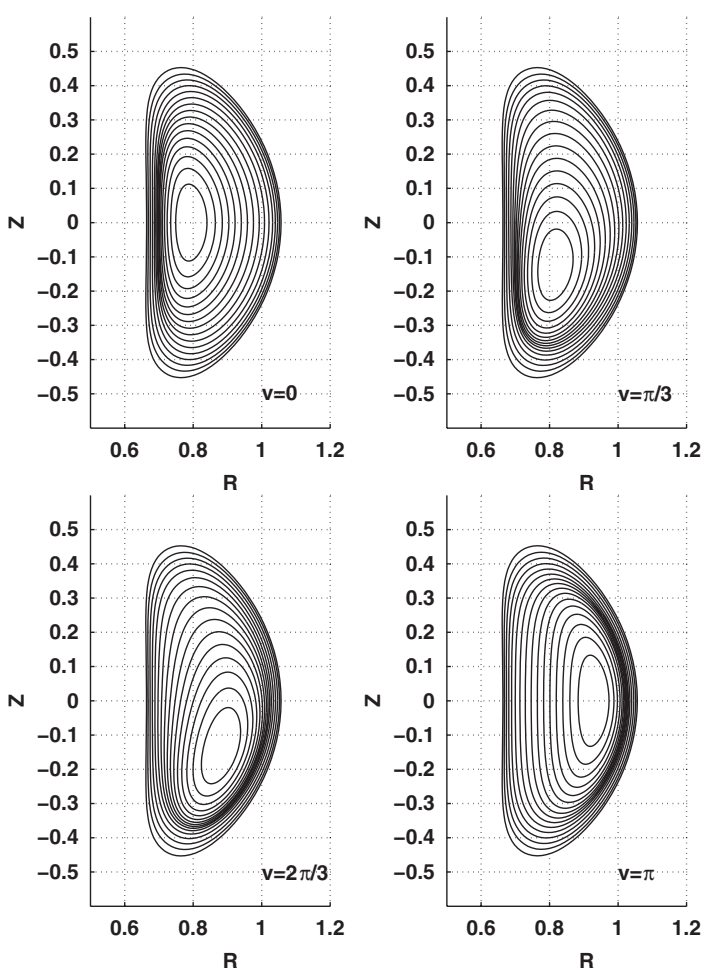

FIG. 2. Toroidal magnetic flux surface contours for the helical equilibrium state branch at four different toroidal cross sections with angles $v=0, \pi / 3,2 \pi / 3$, and $\pi$ in TCV-like axisymmetric boundary geometry with elongation 2.4 , triangularity 0.3 , and rotational transform profile $\iota(s)=0.9+0.2 s-0.8 s^{6}$. The equilibrium energy is $\mu_{0} W_{\text {hel }}=1.454960$.

A bifurcation phenomenon takes place with very flat and extended or hollow $q$ profiles where $q_{\min }$ approaches unity such that two MHD equilibrium states exist. The equilibrium states are calculated using the ANIMEC code [9] (an anisotropic pressure variant of the VMEC code [7]), in which the pressure is prescribed as isotropic. Identical pressure, rotational transform and plasma boundary are imposed. The sole difference is the initial guess provided for the position of the magnetic axis. For the axisymmetric case, the $m=0, n=1$ Fourier components $R_{01}(0)$ and $Z_{01}(0)$ are chosen to vanish. Figure 1 shows the magnetic flux contours on four toroidal cross sections spanning half of the torus. The cross sectional cuts are identical. For this particular example, the rotational transform profile is given by $\iota(s)=0.9+0.2 s-0.8 s^{6}$. However, choosing as an initial guess for the magnetic axis position the Fourier amplitudes $R_{01}(0)=-0.08$ and $Z_{01}(0)=0.10$, we obtain an equilibrium state that has a $3 \mathrm{D}$ helical core as shown in Fig. 2 with converged values of $R_{01}(0)=-0.071$ and $Z_{01}(0)=0.157$. This bifurcated equilibrium state has a slightly lower MHD energy level than that of its axisymmetric fraternal twin. A dominant $m=1, n=1$ structure describes the helical core.

A selected set of $\iota(s)$ profiles are presented in Fig. 3 varying from hollow to very flat, all of which have bifurcated equilibrium states (except the dashed curves). We 


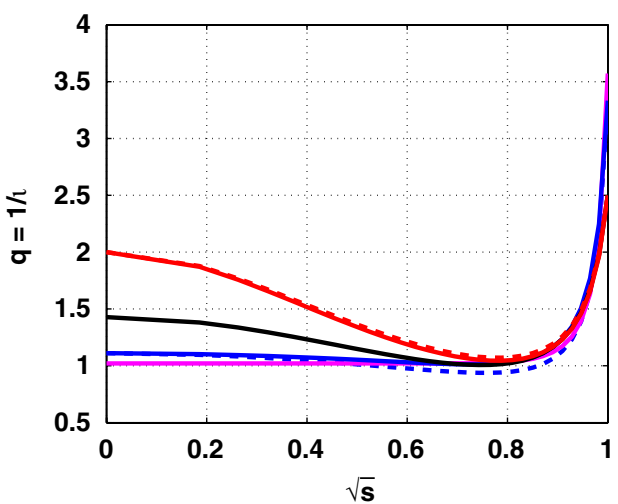

FIG. 3 (color online). A selection of inverse rotational transform profiles that yield bifurcated equilibrium states as a function of $\sqrt{s}$. From top to bottom, the $\iota$ profiles are $0.5+s-$ $1.1 s^{4}, \quad 0.7+0.7 s-s^{4}, 0.9+0.2 s-0.8 s^{6}$, and $0.98-0.7 s^{9}$ (solid curves). The dashed curves correspond to neighboring $\iota$ profiles for which only the axisymmetric branch exists.

explore the operational range in which internal helical structures appear by varying the inverse rotational transform on axis $q_{0}$, that at the edge of the plasma $q_{b}$ and that of the minimum value $q_{\min }$. A good measure of the helical distortion of the magnetic axis are the converged values of the $m=0, n=1$ Fourier amplitudes of $R$ and $Z$ on axis. We first have investigated a sequence of equilibria for which $\iota(s)=0.7+0.7 s-\alpha_{4} s^{4}$ was prescribed, with $\alpha_{4}$ varied between 1.0 and 1.4 and found internal helical structures that become larger as $q_{b}$ decreases. However, as we modify $q_{b}$, we are actually also changing $q_{\min }$, so the relative impact of each variable is not clear. In another sequence of equilibria we maintain both $q_{0}$ and $q_{b}$ fixed and only alter $q_{\min }$. Figure 4 , shows a significant helical distortion of the magnetic axis when $q_{\min } \simeq 1$ that disap-

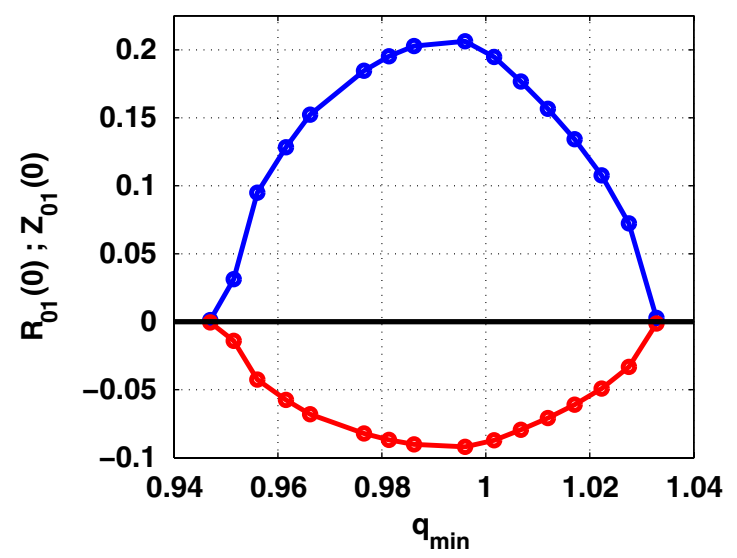

FIG. 4 (color online). The estimate of the helical distortion of the magnetic axis given by the dominant Fourier components $R_{01}(0)$ (bottom curve) and $Z_{01}(0)$ (top curve) at the magnetic axis (corresponding to $m=0, n=1$ ) is shown as a function of $q_{\min }$ for a sequence of equilibria with prescribed $\iota(s)=0.9+\alpha_{1} s-$ $\left(0.6+\alpha_{1}\right) s^{6}$, where $\alpha_{1}$ is varied between 0.16 and 0.32 . In this sequence, $\iota(0)=0.9$ and $\iota(1)=0.3$ are fixed. The horizontal line with zero amplitude constitutes the axisymmetric branch. pears, reducing to the axisymmetric branch, only when $q_{\min }$ exceeds 1.034 or is less than 0.95 . For the same sequence explored in Fig. 4, we present the difference between the energy of the helical state and that of the axisymmetric state in Fig. 5 showing that the helical bifurcated state has lower energy. Nevertheless, the difference in energy between the two equilibrium states is actually very small though the distortion to the equilibrium can be significant as shown in Fig. 4. We would therefore envisage that transitions back and forth between the axisymmetric and helical equilibria could easily take place because no one state has distinctively lower energy (thus is more stable) than the other, though nonideal effects could alter this hypothesis.

Experimental observations in TCV indicate that the pressure profile is flat inside the surface $q=q_{\min }$ with a steep gradient outside [11,12]. Profiles that satisfy these characteristics are plotted in Fig. 6, where we vary $p_{0}$ to generate a finite $\beta$ scan. The largest volume-averaged $\langle\beta\rangle \simeq 3 \%$ was obtained with $p_{0}=4 \times 10^{4}$. Interestingly, the equilibrium state is axisymmetric at low $\beta$ and develops a bifurcated helical solution when $\langle\beta\rangle>0.7 \%$ in Fig. 7. The helical distortion of the magnetic axis approaches a magnitude $\sim 1 / 3$ of the minor radius at $\langle\beta\rangle \simeq$ $3 \%$. It should be noted that helical states with a different $q$ profile have been computed at vanishing $\langle\beta\rangle$. Hence, the dominant factor in the formation of an internal 3D structure is the current profile and the proximity of $q_{\min }$ to unity, though finite $\langle\beta\rangle$ will also contribute.

In summary, a spontaneous bifurcation phenomenon is observed in the computation of tokamak magnetohydrodynamic equilibrium states with a fixed axisymmetric boundary. Under a variety of conditions with either reversed global magnetic shear in the center of the plasma or a very flat inverse rotational transform $q$ profile, two equilibrium states can develop when $0.93 \leq q_{\min } \leq 1.05$. One retains axisymmetry, while the other has a threedimensional helical core similar to a saturated dominantly $m=1, n=1$ internal kink mode. Though the energy

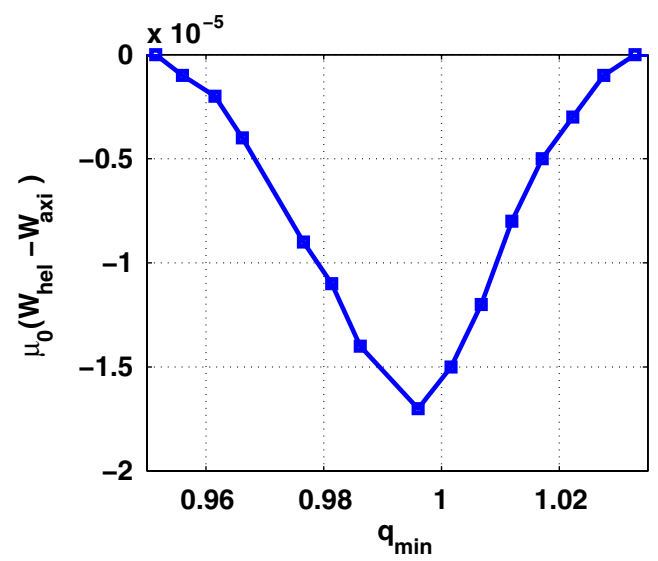

FIG. 5 (color online). The difference between the equilibrium energy of the helical state and the axisymmetric state as a function of $q_{\text {min }}$ for the sequence of equilibria described in Fig. 4. 


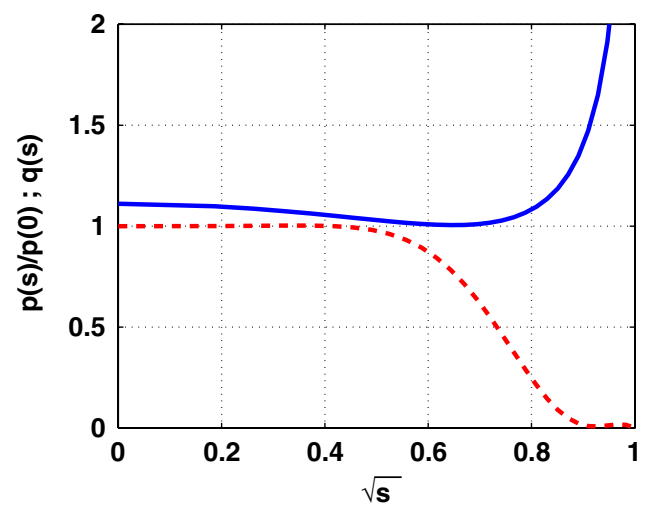

FIG. 6 (color online). The pressure $p(s)=p_{0}(1+$ $\left.0.410227 s^{2}-14.1988 s^{4}+29.6253 s^{6}-22.9512 s^{8}+6.1152 s^{10}\right)$ (lower curve) and the inverse rotational transform $q(s)=(0.9+$ $\left.0.3 s-s^{4}\right)^{-1}$ (upper curve) profiles as a function of $\sqrt{s}$ for the $\langle\beta\rangle$ scan study.

levels of these bifurcated states are very similar, their geometric structures become distinctly different. The helical state can have, just slightly, a lower energy compared with its axiymmetric counterpart. The helical equilibrium states are particularly favored when the radial location of $q_{\min }$ typically exceeds half the enclosed plasma volume for $q_{b}<3-4$. When $q_{\min } \geq 1.06$ or $q_{\min } \leq 0.9$, only an axisymmetric equilibrium is obtained. We therefore predict that the TCV tokamak with large elongation and flat or hollow $q$ profiles (with large shear reversal radius) could acquire a 3D helical core. The existence of these new 3D equilibrium states within axisymmetric tokamak geometry opens new research possibilities, in particular, for explaining the radical change of the sawtooth behavior in highly elongated [11,12] and oval-shaped [23] plasmas, the robustness of the "snake" structures to internal perturbations [24] and the ubiquitous appearance of the ideal mode perturbing MAST discharges [13]. The confinement of $\alpha$ particles in reactor systems in the presence of internal

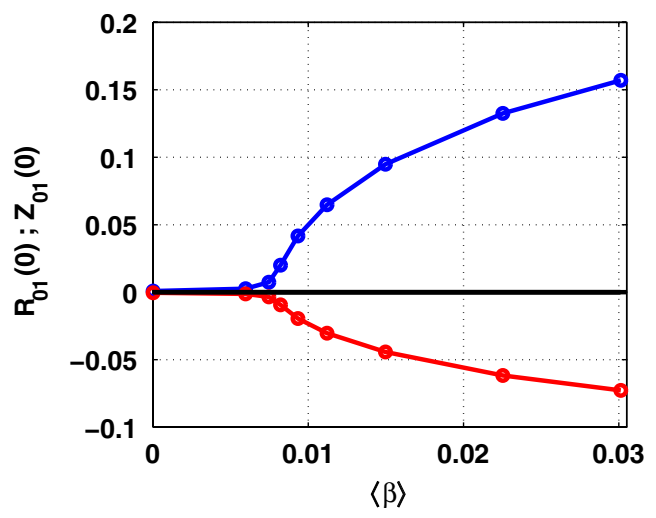

FIG. 7 (color online). The dominant Fourier components $R_{01}(0)$ (bottom curve) and $Z_{01}(0)$ (top curve) at the magnetic axis are plotted as a function of $\langle\beta\rangle$ for a sequence of equilibria with the profiles of Fig. 6 with $q_{\min }=1.005$. The horizontal line with zero amplitude constitutes the axisymmetric branch. helical structures will become a highly relevant issue with implications for the "ITER hybrid scenario" [25].

This work was supported in part by the Swiss National Science Foundation. We wish to thank Professor Francis Troyon for his long-term encouragement and support of this research. We are very grateful and indebted to S.P. Hirshman and for the use of his VMEC code which has made this investigation possible. W. A. C. extends his appreciation to D. Terranova, M. Gobbin, P. Martin, I. Predebon, and L. Marrelli for discussions on helical structures in RFX-mod. We thank B. Duval for carefully revising this manuscript.

*wilfred.cooper@epfl.ch

[1] H. Grad and H. Rubin, in Proceedings of the Second UN Conference on the Peaceful Uses of Atomic Energy (IAEA, Geneva, 1958), p. 190, Vol. 31.

[2] V.D. Shafranov, in Reviews of Modern Physics (Consultants Bureau, New York, 1966), Vol. 2, p. 103.

[3] S. P. Hirshman and J. C. Whitson, Phys. Fluids 26, 3553 (1983).

[4] U. Schwenn, Comput. Phys. Commun. 31, 167 (1984).

[5] A. Bhattacharjee, J. C. Wiley, and R. L. Dewar, Comput. Phys. Commun. 31, 213 (1984).

[6] F. Bauer, O. Betancourt, and P. Garabedian, Magnetohydrodynamic Equilibrium and Stability of Stellarators (Springer-Verlag, New York, 1984).

[7] S.P. Hirshman, W.I. van Rij, and P. Merkel, Comput. Phys. Commun. 43, 143 (1986).

[8] N. Pomphrey (private communication).

[9] W. A. Cooper et al., Comput. Phys. Commun. 180, 1524 (2009).

[10] F. Hofmann et al., Plasma Phys. Controlled Fusion 36, B277 (1994).

[11] H. Reimerdes et al., Plasma Phys. Controlled Fusion 48, 1621 (2006).

[12] Y. Camenen et al., Nucl. Fusion 47, 586 (2007).

[13] I. T. Chapman et al. Nucl. Fusion 50, 045007 (2010).

[14] J. A. Wesson, Plasma Phys. Controlled Fusion 37, A337 (1995).

[15] M.E. Puiatti et al., Plasma Phys. Controlled Fusion 51, 124031 (2009).

[16] W. Park and S. C. Jardin, Phys. Fluids 26, 1871 (1983).

[17] F. L. Waelbroeck, Phys. Fluids B 1, 499 (1989).

[18] L. A. Charlton, R. J. Hastie, and T. C. Hender, Phys. Fluids B 1, 798 (1989).

[19] H. Lütjens and J. F. Luciani, J. Comput. Phys. 227, 6944 (2008).

[20] O. Betancourt, Commun. Pure Appl. Math. 41, 551 (1988).

[21] F. Bauer, O. Betancourt, and P. Garabedian, Proc. Natl. Acad. Sci. U.S.A. 85, 7423 (1988).

[22] P. Garabedian, Proc. Natl. Acad. Sci. U.S.A. 103, 19232 (2006).

[23] E. A. Lazarus et al., Plasma Phys. Controlled Fusion 48, L65 (2006).

[24] A. Weller et al., Phys. Rev. Lett. 59, 2303 (1987).

[25] C. Gormezano et al., Plasma Phys. Controlled Fusion 46, B435 (2004). 